\title{
Costo, volumen, precio y utilidad: dinámica del desempeño financiero industria confecciones infantiles
}

Cost, volume, price, and usefulness: dynamics of the financial performance of the children's apparel industry

Coût, volume, prix et revenu: dynamique de la performance

financière dans $l$ industrie des confections infantiles

Ofelia Gómez Niño ofeliagmz@yahoo.com Unidades tecnológicas de Santander Bucaramanga-Colombia

Magister en Administración de Empresas Universidad Santo Tomás. Unidades tecnológicas de Santander. Dirección de Investigaciones, Grupo de Investigación en Ciencias Socioeconómicasy Empresariales (GICSE). Docente investigadora.

Artículo de investigación científica y tecnológica Según Clasificación Colciencias

Fecha de recepción: $13 / 03 / 2012$

Fecha de corrección: $08 / 04 / 2012$

Fecha de aprobación: 20/05/2012

\section{Resumen}

Este artículo resume los resultados de investigación en 52 empresas de la industria de confección infantil en Bucaramanga. Es un estudio descriptivo, que permitió establecer las relaciones existentes entre procesos de producción, sistemas de costeo y estrategias, en función del costo, volumen y utilidad, en la dinámica del desempeño financiero de estas organizaciones. Los hallazgos, evidencia la relación entre producción, costos y estrategias que son las variables influyentes del desempeño financiero, en esta industria. Asimismo, la relación costo-volumen-utilidad contribuyen en el análisis, porque el precio se fija en función del costo, los costos según los recursos consumidos y volumen de producción, y el margen de contribución resulta de la diferencia entre precio y costo, que se equipara para cubrir los gastos operacionales e impuestos, y proporcionar utilidad. Además, las estrategias se enfocan hacia la productividad, sustentada en la capacitación, la tecnología y asociatividad, para fortalecer el desempeño financiero y la sostenibilidad en el mercado.

Palabras clave: costo, volumen, utilidad, estrategia, industria confecciones, desempeño financiero 
Cost, volume, price, and usefulness: dynamics of the financial performance of the children's apparel industry

Costo, volumen, precio y utilidad: dinámica

del desempeño financiero industria confecciones infantiles

Coût, volume, prix et revenu: dynamique de la performance financière dans l'industrie des confections infantiles

\section{Abstract}

This article summarizes the results of an investigation into 52 companies from the children's garment industry in Bucaramanga - Colombia. It is a descriptive study, which allows establishing the relationships among the production processes, costing systems, and strategies regarding the cost, volume and utility in the dynamics of the financial performance of these organizations. The findings prove the connection among production, costs, and strategies, which are the variables that influence financial performance in this industry. Likewise, the cost-volume-utility relationship supports this analysis because price is directly related to cost and these costs, according to the resources consumed and the volume of production, along with the margin of contribution is the difference between price and cost, which covers the operational expenses and taxes and yields profit. Furthermore, the strategy focuses on productivity, which is supported by training, technology, and associativity to strengthen the financial performance and sustainability of the market.

Keywords: cost, volume, usefulness, strategy, apparel industry, financial performance.

\section{Coût, volume, prix et revenu: dynamique de la performance financière dans $l^{\prime}$ industrie des confections infantiles}

Costo, volumen, precio y utilidad: dinámica

del desempeño financiero industria

confecciones infantiles

Cost, volume, price, and usefulness:
dynamics of the financial performance of the
children's apparel industry

Résumée

Cet article résume les résultats de recherche à 52 entreprises de l'industrie de confections infantiles à Bucaramanga. C'est une étude descriptive, qui a permis d'établir les relations existantes entre le processus de production, les systèmes de détermination de coût et les stratégies, en fonction du coût, du volume et du revenu, dans la dynamique de la performance financière de ces organisations. Les résultats mettent en évidence la relation entre production, coûts et stratégies qui sont les variables influentes de la performance financière dans cette industrie. De même, la relation coût-volume-revenu contribue à l'analyse, parce que le prix se fixe en fonction du coût, les coûts selon les ressources consommées et le volume de production, et la marge de contribution résulte de la différence entre prix et coût, qui se compare pour couvrir les coûts opérationnels et les impôts, et donne le revenu. En outre, les stratégies se visent vers la productivité, soutenue par la formation, la technologie et l'associativité, pour renforcer la performance financière et la soutenabilité dans le marché.

Mots clef: coût, volume, revenu, stratégie, industrie confections, performance financière. 


\section{Costo, volumen, precio y utilidad: dinámica del desempeño financiero industria confecciones infantiles}

\section{Introducción}

La tendencia de los mercados y la dinámica económica global, exige a las empresas definir modelos de administración eficientes. Por lo tanto, la industria de la confección infantil reconsidera la formulación de estrategias en función de los costos e ingresos, mediante la optimización de recursos para flexibilizar las relaciones del costo-volumen-utilidad. Dentro de este marco, Porter (2007, p. 51) menciona que en una economía global el éxito o fracaso de los negocios depende de la capacidad competitiva con la que participen en los mercados, capacidad que se mide por factores relacionados con la estrategia de diferenciación, liderazgo global en costos, enfoque o concentración. La industria de confección textil encara serias dificultades en el desarrollo de los negocios y por ende, el logro de los objetivos básicos financieros y como consecuencia de los tratados de libre comercio, han llegado nuevos competidores con mayor tecnología, condición que deja en desventaja a los pequeños empresarios y por lo tanto, se reduce la posibilidad de tener negocios rentables.

La productividad de un país, industria o empresa se determina por la cantidad de productos 0 servicios que genere con los mínimos insumos, es decir, que la optimización de los recursos produce una elevación de los niveles de eficiencia, expresada a través de procesos altamente productivos. De ahí que el departamento de Santander se caracterice por ser una región productiva y se destaque por la ubicación geográfica y fuentes de riqueza natural. Datos del DANE muestran un crecimiento económico favorable de $5,8 \%$ en el período 2000 a 2008; el ingreso bruto percápita logró niveles aceptables con 7.698 dólares, fue el tercero entre 32 departamentos colombianos, y el segundo entre las cinco mejores economías del país. Por lo tanto, la competitividad regional se soporta en crecimiento empresarial, educación, desarrollo tecnológico, fomento del agro y fortalecimiento del sector productivo, factores que evidencian el mejoramiento en los indicadores de desempeño económico y posiciona a este departamento en el cuarto lugar de la economía nacional, después de Cundinamarca, Antioquia y Valle. Según datos de la Cámara de Comercio (2010), esta industria generó la mayor participación con el 31,3\%, seguido por la actividad de servicios $29,2 \%$ y comercio $11,2 \%$ (Cámara de Comercio, 2010).
En consecuencia, el sector industrial de confección textil está integrado por gran cantidad de micro y pequeñas empresas, constituidas por el núcleo familiar, que impulsa la generación de empleo y fuentes de trabajo sobre todo para mujeres cabeza de familia, contribuyendo a la solución de problemas sociales que afectan a los habitantes de este departamento. Por esta razón se consideró relevante que dentro de los objetivos de este trabajo de investigación se contemplara variables como costos, volumen y utilidad en función de analizar sus relaciones y establecer estrategias orientadas con el aprovechamiento de los recursos con el fin de minimizar costos, aumentar la producción, fijar precios de venta competitivos y lograr las expectativas de los empresarios. Aunque estas empresas posean limitaciones en el desarrollo de la actividad económica, son reconocidas en el entorno regional, nacional e internacional, por ofrecer productos con atributos de diferenciación, calidad y costos totales bajos.

El análisis de las relaciones entre las variables observadas como el costo de producción, precio de venta y margen de contribución, permitió establecer que estas empresas utilizan como estrategia aumentar la eficiencia para elevar el nivel de producción y minimizar los costos de los productos, de modo que los precios de venta se ajusten a las condiciones del mercado y la competencia, y se conviertan en negocios competitivos para lograr posicionarse en el mercado.

\section{Metodología}

El estudio fue descriptivo. Méndez (2005, p.136) plantea que una investigación es descriptiva cuando se ocupa de detallar las características que identifican los diferentes elementos y componentes y su interrelación. Se analizaron 52 empresas de confección infantil de Bucaramanga, y la observación se centró en la descripción de la estructura y dinámica de las empresas, costos de producción, precios de venta, margen de utilidad, volumen y medios de producción, así como los recursos vinculados con las operaciones. La información recuperada permitió caracterizar a estas empresas e identificar las necesidades que dio paso a una segunda fase de la investigación. De igual forma fue posible analizar las relaciones entre las variables costo-volumen y utilidad en función del precio y las estrategias, con 
el fin de evidenciar la productividad y competitividad de estas organizaciones.

Población y muestra. La población estuvo representada por 224 empresas de confección infantil, registrada y renovada en la Cámara de Comercio de Bucaramanga, año 2010; se tomaron 52 como muestra, este muestreo fue no aleatorio por juicio y conveniencia, teniendo en cuenta en la selección, ubicación geográfica de los principales puntos de concentración productiva.

El número de empresas a observar se determinó con el siguiente procedimiento (Martínez, 2005, p.390 y 392).

$$
\begin{aligned}
& \text { Formula: } n=\frac{n_{o}}{1+n_{o} / N} \\
& \mathrm{~N}=\text { Población } \\
& \mathrm{n}=\text { Tamaño de la muestra } \\
& \mathrm{n}_{\mathrm{o}}=\text { Primera aproximación al tamaño de la } \\
& \text { muestra }
\end{aligned}
$$$$
Z=\text { Nivel de confianza }
$$$$
\mathrm{P}=\text { Proporción de elementos de la población que }
$$
tienen la característica que se está examinando

$Q=$ Proporción de elementos de la población que no tienen la característica que se está examinando

\section{$d=$ Margen de error}

Para desarrollar la fórmula antes expuesta, es necesario tener el valor de $n_{0}$ que se obtiene a partir de la fórmula:

$$
n=\frac{Z^{2} P Q}{d^{2}}
$$

Cuando no se conoce la proporción poblacional se puede tomar el valor de $\mathrm{P}=0,5$ y por lo tanto el de $Q=0,5$, lo que hace que el tamaño de la muestra sea lo suficientemente grande para garantizar como mínimo la precisión requerida en el estudio.

Para el caso de las fábricas de confecciones infantiles de Bucaramanga, la información con la que se determinó la muestra fue la siguiente:

$\mathrm{N}=224$ empresas (fábricas registradas y renovadas período 2009, Cámara de Comercio de Bucaramanga)
$Z=$ Nivel de confianza se determina el $90 \%$

(según tabla, 1,645)

$\mathrm{d}=$ Margen de error del muestreo se estipula en un $10 \%(0,1)$

$\mathrm{P}=$ Proporción de elementos de la poblacional que tiene las características $(0,5)$

$Q=$ Proporción de elementos de la poblacional que no tienen las características $(0,5)$

La primera aproximación a la muestra es de 67,650625 y a partir de este valor se determina la muestra que corresponde a:

$$
n_{0}=\frac{(1,645)^{2}(0.5)(0,5)}{(0,1)^{2}}
$$

Fuentes de información. El estudio se soportó en fuentes primarias y secundarias.

Fuentes primarias. Para la recolección de información se diseñó una encuesta con 32 preguntas relacionadas con las principales variables a estudiar. La aplicación del instrumento se realizó mediante visita a las empresas y la información fue suministrada directamente por los empresarios, dueños o gerentes.

Fuentes secundarias. Se consultaron documentos sobre estudios realizados en las empresas del sector, se revisó la base de datos en la Cámara de Comercio de Bucaramanga, se buscó información de gremios y se tomó como apoyo material de artículos, libros, tesis de grado de maestrías y doctorados, entre otros.

Análisis de la información. La información suministrada en cada pregunta se tabuló y graficó en hojas de Excel, la interpretación y análisis se soportó en herramientas estadísticas en función de los objetivos trazados.

\section{Resultados}

\subsection{Contextualización de la industria de confección textil}

En Colombia, la industria de confecciones ha sido un importante reactivador de la economía nacional y se considera un generador de empleo por aportar $11 \%$ del total de la industria. De la misma manera la producción se considera un indicador de reacción de la economía, el sector tiene una participación del 3,3\% del total de la industria, cifra influyente como aporte al PIB na- 
cional (ANDI. Asociación Nacional de Industriales de Colombia, 2009).

Para objeto de la investigación, es conveniente anotar que pertenecen a la industria de las confecciones, aquella unidad productiva cuya principal actividad es la transformación de materia prima en una prenda de vestir o un artículo confeccionado. En tal sentido estas empresas producen diferentes líneas de productos y buscan la especialidad en la fabricación de prendas de hombre, dama, niño, niña y bebé.

De otra parte, se logró establecer que en el año 2010, estaban inscrita en el Registro Mercantil de Bucaramanga 53,354 empresas de las cuales 7164 corresponden a la actividad industrial, en las que se ubican las de confección infantil, segmento de la industria que le aporta al desarrollo de la economía regional y contribuye en la solución de problemas sociales, como es el desempleo, (Cámara de Comercio, 2010). De igual manera es importante mencionar que de las empresas que participaron en la investigación, $88 \%$ son micro y pequeñas empresas, esta clasificación se soporta en el número de trabajadores contratados directamente.

Cabe señalar que esta industria hace un aporte representativo a la generación de empleo, proporcionado por micros y pequeñas empresas. De igual forma se destaca que a pesar de que estas organizaciones son un pilar fundamental para la economía de la región, no están muy tecnificadas, cuentan con maquinaria básica y los procesos son desarrollados en gran medida de manera artesanal, aspectos que aportan gran valor al producto en los procesos de producción, por el ingenio y creatividad que aplica el recurso humano, atributos que le permiten participación en el mercado, colocando el sello de calidad y diferenciación en prendas de vestir para la población infantil.

También la fabricación de estas prendas ha sido una tradición en la región, y ha pasado de generación en generación, prevaleciendo esta actividad en grupos familiares, labor que se refleja en los diferentes municipios del departamento, entre los que se destacan las bordadoras de Zapatoca, armadoras de Floridablanca, cortadoras de Piedecuesta, que han contribuido con la ampliación de la capacidad instalada de las empresas dedicadas a esta actividad.

Otro aspecto de gran relevancia en esta industria manufacturera de la región, es lo relacio- nado con los eventos organizados por gremios y organismo gubernamentales cuyo propósito es el de propiciar los escenarios para la construcción de imagen y así fortalecer el posicionamiento de los producto y brindar mayores oportunidades a quienes participan positivamente en impulsar a este sector, en los que:

la especialización en la moda infantil, la calidad de los productos, su trayectoria exportadora y la existencia de un escenario comercial como EIMI, les ha permitido a las empresas tener contacto con compradores internacionales para hacer negocios y dinamizar las relaciones comerciales exitosas a largo plazo. Estas son algunas claves de la evolución del sector (Trujillo, 2010).

\subsection{Productividad y competitividad, opción estratégica de la industria}

Ahora es oportuno, establecer aquello que define la productividad y competitividad de la industria; estos dos términos guardan una estrecha relación: el primero se refiere al uso de los recursos de producción en términos de eficiencia, es decir, optimizarlos para lograr el máximo resultado (volumen de producción alto, al menor costo, conservando la calidad); esto implica trabajar aplicando métodos que permitan reducir el desperdicio de los recursos y controlar los procesos para evitar que se desmejore la calidad. El segundo consiste en participar en el mercado con productos, bienes o servicios diferenciados; esta diferenciación puede estar dada por atributos como especificaciones de diseño y materiales, innovación y desarrollo, atención a las necesidades de los consumidores, costo y calidad, entre otros. Para ser productivos en realidad se deben evitar los productos defectuosos, pues los defectos no salen gratis, alguien los hace, y se le paga por hacerlos, por lo tanto afecta el costo y la calidad y la mala calidad disminuye la productividad en una línea de producción (Deming, 2008, p.9).

Bajo esta óptica, la industria de confecciones infantiles de Santander en su trayectoria, ha logrado posicionarse en el mercado nacional e internacional, a través de un gran esfuerzo por diversificar la oferta de líneas de productos innovadores con alto componente artesanal, y a costos relativamente ajustados a los de la competencia. En este orden de ideas, se considera significativo resaltar el compromiso de los gremios y entes gubernamentales con el posicionamiento de esta industria en el mercado nacional e internacional, a 
través de la organización de eventos como ruedas de negocios, ferias, programas de capacitación en diversas áreas, de manera que concurran los empresarios y compradores a estos escenarios.

De igual forma, las empresas buscan definir sus propias estrategias para fortalecer los procesos, uso de recursos, aprovechamiento del mercado y generación de canales de distribución, todo ello con el fin de mejorar el desempeño financiero que se establece a partir de la relación del costo, precio de venta y la utilidad. En esta dinámica, los negocios en el mundo actual como los de confecciones infantiles, requieren ajustar las estructuras y repensar los procesos para orientarlos a nuevas formas de realizar el trabajo, aplicación de tecnología para hacer que los negocios se vuelvan competitivos y de ahí la relevancia en definir estrategias acertadas. Acá las organizaciones deben concentrar el esfuerzo en hacer que los negocios logren crecer, generar utilidades y permanecer en el mercado; por lo tanto debe ser claro hacia dónde van, es decir, haber definido su direccionamiento estratégico, integrado por los principios corporativos, la misión y visión de la organización, así como el compromiso social que de ellas se derivan (Amaya, 2005, p. 35).

Desde esta perspectiva, se propone formular estrategias a partir de un contexto general en el que se precisan los factores que soportan la estrategia a aplicar. Las empresas deben ser creativas en el diseño de estrategias, de manera que estas permitan el alcance de metas y propósitos organizacionales, en lo posible enfocarlas a elevar el volumen de producción, reducir costos sin afectar la calidad, fijar precios competitivos para incrementar los ingresos y generar rentabilidad.

De este modo, Porter (2007, p. 13) propone que al formular estrategias, es indispensable:

Examinar los cuatro factores que determinan los límites de lo que la compañía podrá lograr. Sus puntos fuertes y débiles representan su perfil de activos y sus habilidades en relación con la competencia como recursos, situación tecnológica, identificación de marca, entre otras cosas. Los valores personales son los motivos y necesidades de los principales ejecutivos y de otros empleados que se encargan de la estrategia escogida. Los puntos fuertes y débiles, combinados con los valores, determinan los límites internos de la estrategia competitiva que una compañía puede adoptar exitosamente.
Aunque la industria de confecciones infantiles de Bucaramanga, ha ganado prestigio y reconocimiento, aún tiene serias limitaciones para alcanzar el ideal en la función financiera. Sin embargo, un buen análisis a esta industria permite avanzar en los procesos de mejoramiento, en elementos puntuales que afectan el desempeño. Es preciso ubicar a estas organizaciones en un contexto moderno, en el que la economía propone mejores prácticas administrativas, financieras y sociales, para lograr la sostenibilidad y crecimiento de una unidad de negocio. Es así, como desde los ambientes de alta dirección se formulan estrategias que se direccionan a impulsar a estas empresas en la búsqueda de la eficiencia; por lo tanto, se debe identificar las variables claves para ser contempladas en la definición de la estrategia, como se muestra en la Figura 1.

\section{Figura 1. Contexto de la estrategia competitiva}

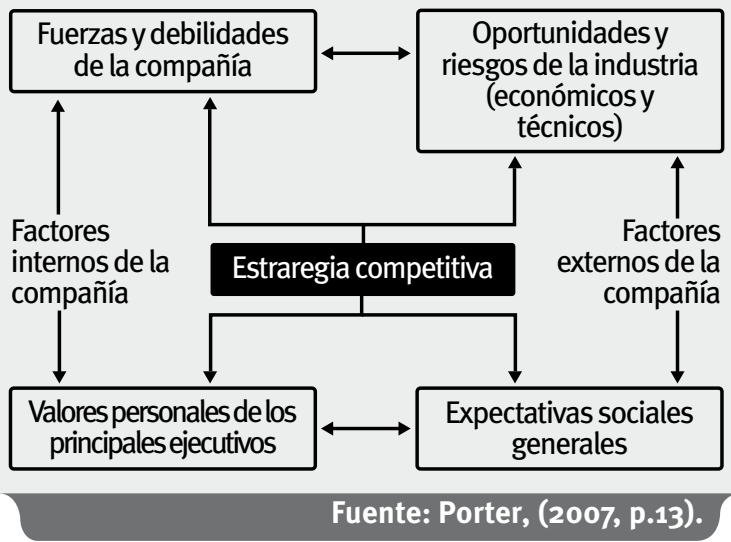

A propósito del análisis realizado en las 52 empresas del sector industrial de confección textil infantil que representan el $23 \%$ del total de la población, se logró establecer que las principales debilidades se relacionan con las estructuras de costos, capacidad instalada, organización administrativa, entre otros. Sin embargo, no todo es negativo porque poseen fortalezas en el aprendizaje de la dinámica de estos negocios, aspectos que les ha permitido el reconocimiento en el mercado regional, nacional e internacional, producto de la innovación, variedad de diseños, componente artesanal, materiales e insumos utilizados en la producción, diversificación en las líneas de productos fabricados y la calidad de los mismos, factores determinantes en la decisión de compra, lo cual fomenta las exportaciones y fortalece el mercado global (Cámara de Comercio - Bucaramanga, 2010). 
Ahora bien, estas empresas enfocan las estrategias hacia el fortalecimiento de la tecnología, mejoramiento de la mano de obra, cultura de asociatividad, aspectos que se promueven a través de programas de capacitación, inversión en tecnología, conformación de clústeres, participación en agremiaciones, elementos que las impulsan al posicionamiento con el fin de alcanzar los objetivos financieros (Gráfica 1).

\section{Gráfica 1. Estrategias para mejorar la productividad}

Estrategias de competitivad

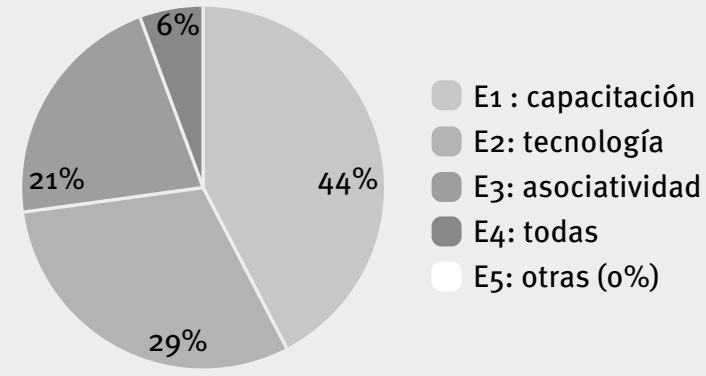

Fuente: Gómez, 0. (2012) con información empresas estudiadas, 2010.

De igual forma, este estudio muestra que el $44 \%$ de las empresas que participaron en la investigación, tiene como principal variable para formular estrategias de productividad, lo relacionado con la capacitación del recurso humano y el $29 \%$ de las mismas, consideran que la tecnología es de gran relevancia, debido a que estas contribuyen con la productividad, calidad y diferenciación de los productos en las diversas líneas de producción. Por esta razón, para alcanzar los objetivos básicos financieros, se requiere enfocar a la organización hacia el direccionamiento estratégico, porque este servirá de marco de referencia para el análisis de la situación actual de las compañías, en el entorno interno y externo, para poder responder a las preguntas: ¿Dónde estábamos? ¿Dónde estamos hoy? (Amaya, 2005, p. 34). De ahí, que las estrategias que fijan estas empresas no sean ruedas sueltas, ellas deben responder a necesidades identificadas mediante técnicas y procedimientos, de manera que guarde coherencia con el modelo de administración adoptado por esta industria.

\subsection{Costo de producción y fijación de precios}

Para abordar los costos, se requiere contextualizar las organizaciones, generalmente de ellas derivan su naturaleza. Los costos de producción son propios de la industria; es en este contexto donde se generan como resultado de la integración de insumos a un proceso de transformación, utilizando un sistema de producción previamente estructurado; este permite la acumulación de los importes económicos, que luego se convierten en el costo de fabricación, bien sea de una unidad o lote de productos y este valor es la base sobre la cual se fija el precio de venta y margen de contribución.

Al comenzar a relacionar estas variables se considera oportuno tener en cuenta a Polimeni et al. (2005, p. 11) que define el costo como:
El "valor" sacrificado para adquirir bienes o servicios, que se mide en dólares me- diante la reducción de activos o al incurrir en pasivos, en el momento en el que se obtienen los beneficios. En el momento de la adquisición, el costo en que se incurre es para lograr beneficios presentes o futuros. Cuando se utilizan estos beneficios, los costos se convierten en gastos. Un gasto se define como un costo que ha producido un beneficio y ha expirado. Los costos no expirados que pueden dar beneficios futu- ros se clasifican como activos.

Los gastos se confrontan con los ingresos, para determinar la utilidad o la pérdida netas de un período. El ingreso se define como el precio de los productos vendidos o de los servicios prestados. En determinadas circunstancias, los bienes o servicios comprados se convierten en algo sin valor, sin haber prestado ningún beneficio. Estos costos se denominan pérdidas y se presentan en el estado de ingresos como una deducción de los ingresos, en el período que ocurrió la disminución en el valor.

Por supuesto que la industria de confecciones incurre en costos cuando realiza la compra de los bienes e insumos necesarios para producir los productos. Además requiere de mano de obra calificada que puede contratarla directamente, o subcontratarla con talleres satélites. De otra parte, para la producción utiliza maquinaria, equipos, planta física, tecnología, elementos que se pueden adquirir o tomar en arriendo y por lo tanto también requiere de un sacrifico económico. Desde esta perspectiva, la integración de los diversos componentes en términos de valor, se convierte en el costo de producción, integrados al producto, que al ser vendido, se transforma en ingresos, y de la relación de estas dos variables, se obtiene la utilidad o pérdida en un período, resultados que afecta negativa o positivamente el patrimonio de la compañía. 
Acá conviene anotar, que las empresas se constituyen con fines económicos, aunque tienen una función social, los inversionistas esperan rentabilidad para incrementar la riqueza; por lo tanto estas se convierten en factor de desarrollo de una región o país y a su vez son fuente de trabajo e ingresos para las comunidades relacionadas. Asimismo, el desarrollo de la actividad, genera transacciones que deben ser sistematizadas para llevar a cabo el control del negocio, información sintetizada en los estados financieros. Barker (2005, p. 91) expone, que los informes financieros "agrupa ingresos y gastos, para los propósitos de una eficiente expresión del desempeño financiero. En particular, si transacciones y hechos diferentes dan origen a ingresos y gastos con propiedades informativas similares, entonces, estos pueden agruparse como componente del resultado integral".

Con todo lo anterior, vale la pena señalar que la productividad de la industria se deriva en gran parte de la producción, de ahí que, Meléndez (2004, p. 15) plantea la dinámica de la gestión de la producción, como un proceso de transformación, que integra una serie de componentes, los cuales proporcionan los medios para convertir unos insumos en productos, bienes o servicios. Por lo tanto, en la medida que se logre optimizar esos medios e insumos, es posible elevar los niveles de productividad y consecuentemente, obtener costos razonables, fijar precios de venta competitivos y mantener el margen de utilidad, acorde con los fines de la empresa (Figura 2).

\section{Figura 2. Proceso de producción}

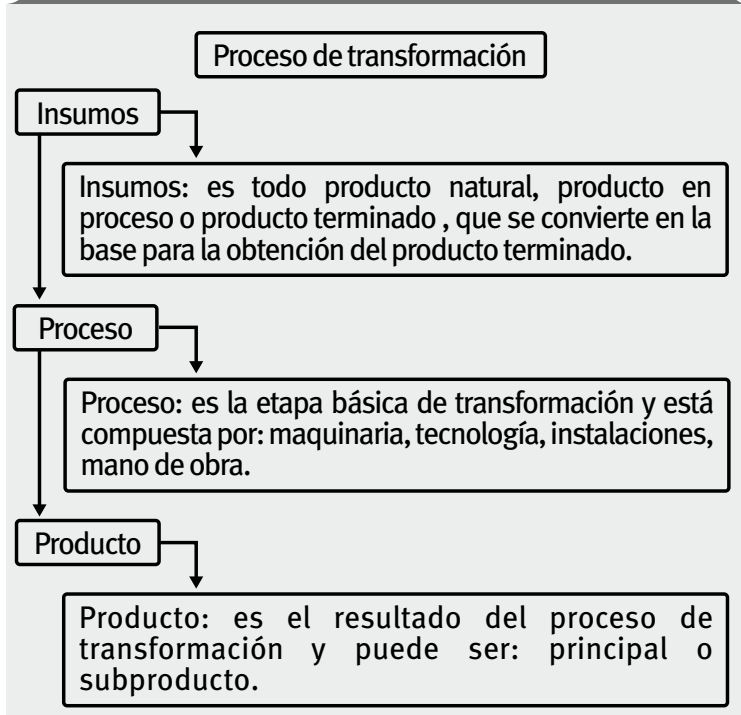

Fuente: Meléndez, H. (2004, p.15).
Bajo este referente, tiene sentido dar una mirada a la forma como las empresas de confecciones infantiles de Bucaramanga llevan a cabo los procesos productivos y la administración de los costos relacionados con los mismos. Aquí es oportuno aclarar que en la observación de las 52 empresas se identificó que el $88 \%$ de ellas, se clasifican como micro y pequeñas empresas, con limitaciones en capacidad instalada, entendida esta como las posibilidades que tiene la empresa de producir las unidades necesarias para cumplir los requerimientos de los clientes; en ella se contempla la planta física, la maquinaria, la tecnología, el recurso humano y los recursos económicos. En relación con la capacidad, estas empresas trabajan con una capacidad práctica (Sinisterra 2006, p.178) entendida como "el volumen máximo de producción que se puede lograr, teniendo en cuenta aspectos que pueden originar reducciones en el mismo, como daños en la maquinaria; también se conoce como capacidad real y esta representa $80 \%$ de la capacidad teórica, que es del $100 \%$ ".

Como optimizar los recursos de producción aumenta la eficiencia y como consecuencia se incrementa la productividad, conviene señalar que a partir de estas relaciones se establece el costo de producción. Es así como Faga et al. (2007, p. 12) consideran que el costo es el "sacrificio que se debe realizar para alcanzar un objetivo, cualquiera que sea este. Desde el punto de vista económico en un sentido más estricto, costo es el insumo de determinados elementos valorizables económicamente, aplicado a lograr un objetivo, también económico". En el caso de la industria de confección textil, y a partir de lo observado, se puede decir que el costeo de los productos fabricados se sustenta en la experiencia, aunque integran al producto el costo de materiales e insumos, mano de obra directa y otros costos generales de fabricación y no evidencia un sistema de costos previamente estructurado.

Si bien es cierto, que esta industria fija los precios de venta en función del costo y de la competencia, es relevante tener especial cuidado en la determinación, deben aplicar métodos y técnicas que faciliten una metodología de costos acorde con la realidad económica y condiciones en las que operan estas empresas, de manera que se equiparen a la competencia, para no quedar en desventaja en cuanto al precio. Desde esta óptica, la gestión de costos se convierte en factor determinante de competitividad, Ramírez (2008, p.69) plantea que: 
Para que los administradores decidan cuánto cobrar por un producto o servicio, o cómo hacerle frente a la competencia de otras empresas nacionales o extranjeras, tienen que hacer una estimación razonable de los recursos que se requieren y se consumen para elaborar un producto en particular. Cuando había poca o nula competencia era suficiente tener un sistema de costeo más o menos exacto para un producto o grupo de productos. Actualmente se ha demostrado que los competidores extranjeros están adelante de los productores nacionales, ya que tienen sistemas de costeo más exactos y confiables para tomar decisiones y diseñar estrategias.

Bajo esta premisa, la industria de confecciones infantiles de Bucaramanga, consideran que el costo de producción es la piedra angular para edificar la estructura de costos, en la que el volumen de producción, el precio de venta y el margen de contribución se relacionan para generar reportes financieros, de los cuales se deriven indicadores de medición del desempeño financiero, con el fin de evaluar las estrategias de gestión de costos y fijación de precios (Gráfica 2).

\section{Gráfica 2. Costos la base del precio}

Costos promedio de producción

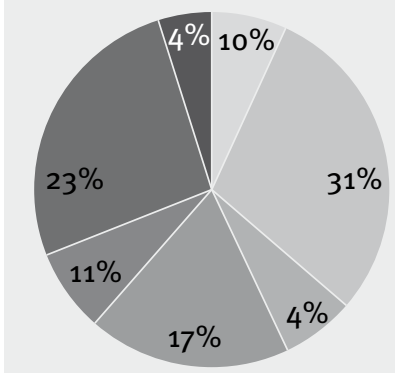

CR: Costos reales

CE: costos estimados

CP: competencia

CRE: realesy estimados

CRC: realesy competencia

CEC: estimadosy competencia

TA: todos los anteriores

Fuente: Gómez, O. (2012) con información empresas estudiadas, 2010.

A todo esto, se añaden los tratados de libre comercio firmados en Colombia, y por esta razón las micro y pequeñas empresas de confecciones se enfrentan a grandes competidores en el mercado; de otro lado en la oferta y la demanda de los productos en este sector de la industria, influye significativamente el precio, que se fija en función del costo y la competencia, indicador que permite poner especial atención en la administración y definición de las estructuras de costos, con el fin de lograr el máximo de eficiencia de los recursos para poder alcanzar costos totales bajos y de esa forma mantener precios equitativos en el mercado. Por cierto, $31 \%$ de las empresas industriales de confección infantil observadas fija el precio de venta soportado en costos estimados y el $23 \%$ lo realiza fundamentados en costos estimados y la competencia.

Cabe anotar, que dos de los aspectos que contribuyen con el posicionamiento de los productos en el mercado, son los relacionados con la diferenciación y el bajo costo, todo ello en la dinámica de la innovación en valor. Por esta razón, Kim et al. (2005, p. 23) propone, que:

El valor para la compañía se genera a partir del precio y la estructura de costos con la que ha fijado dicho precio; por lo tanto sólo existe generación de valor cuando el sistema integral de utilidad, precio y actividades de costos de la compañía están debidamente alineado. Por otra parte, innovaciones como las que ocurre con el producto se pueden lograr al nivel de un subsistema sin afectar la estrategia general de la compañía. Por ejemplo, una innovación en el proceso de producción puede reducir la estructura de costos de la compañía y fortalecer su estrategia existente de liderazgo en costos, sin modificar su propuesta de utilidad.

Respecto a esta posición, la industria de las confecciones infantiles focalizan las estrategias para sustentar el valor en innovación en moda, especificaciones y calidad de las materias primas, mano de obra calificada, alto componente artesanal, oportunidad en la entrega de los pedidos, servicio al cliente, diversificación de líneas, especificación y calidad de los productos y costos razonables. Vale la pena resaltar que estos atributos se convierten en factores estimulantes de la decisión de compra de clientes y consumidores; al mismo tiempo impulsan el volumen de venta e incremento en las utilidades de la compañía.

\subsection{Margen de contribución en función de la utilidad}

En el escenario de las organizaciones con fines económicos, prima ante cualquier situación la búsqueda de riqueza de manera que retribuya la inversión. Sin embargo, como se mencionó antes, las empresas también tienen un compromiso social, condición que debe ser contemplada en la administración de los negocios. Acá se va a analizar lo correspondiente a la función básica financiera, que parte del logro de los objetivos y propósitos empresariales. Una forma de analizar financieramente la empresa es a partir de los resultados de las operaciones en función del desempeño; por lo tanto, 
la relación de las variables costo-volumen-utilidad (CVU) se convierte en un indicador de evaluación. Horngren (2007, p. 60) menciona que esta relación "examina el comportamiento de los ingresos totales, de los costos totales, y de la utilidad operativa a medida que ocurren cambios en el nivel de producción, el precio de venta, el costo variable unitario o los costos fijos de un producto". Estas relaciones y cambios ameritan ser analizados porque define la cantidad de unidades a vender y el precio de venta para sufragar los costos y generar utilidades, además, contribuye con la planeación y análisis del punto de equilibrio.

Después de todo esto, es necesario que las organizaciones se orienten a dar soluciones a problemas propios de la sociedad y a la generación de riqueza para quienes asumen el riesgo de invertir un capital en la construcción de unidades productivas. Aquí se destaca que en el desarrollo de los negocios es factor transcendental las estrategias que aplique la administración para dinamizar la generación de valor para los clientes internos y externos, que permite generar valor agregado e impulsar el crecimiento organizacional y alcanzar la maximización de la riqueza. De este modo, León (1999, p.2) define la función financiera de la empresa teniendo en cuenta que:

La operación de las empresas implica llevar a cabo una serie de actividades o funciones que en términos generales podemos agrupar en cuatro categorías: mercadeo, producción, recursos humanos y finanzas. Ello no significa que siempre deban existir departamentos o áreas con estos nombres o que en el primer renglón jerárquico no puedan haber más de cuatro áreas básicas, pues el diseño de la estructura organizacional depende de aspectos tales como el tamaño y tipo de actividad que desarrolle la empresa, su entorno, sus objetivos y estrategias, entre otros factores, lo cual determina la importancia relativa de las diferentes funciones.

En el caso de las empresas de confecciones, que participaron en la investigación al ser micro y pequeñas, muestran que la organización administrativa es un poco incipiente, por lo tanto no son muy visibles sus áreas funcionales. Es así como dentro de la dinámica de los negocios, es clave establecer la relación costo-volumen-utilidad, con el fin analizar lo relacionado con el punto de equilibrio, entendido este, como el punto en el que los ingresos se igualan a los costos, es decir la empresa vende lo suficiente para cubrir los costos fijos y los variables en relación con los ingresos; en términos de ganancia es el punto cero, donde no existe utilidad pero tampoco pérdida. Estos tres elemento poseen una estrecha relación y son fundamentales en la planeación, ellos están íntimamente relacionados y por lo tanto conviene analizar cómo es esta relación, con el fin de poder tomar decisiones adecuadas en los negocios (Hargadon, 2003, p. 295).

En este orden de ideas, cabe precisar que en las empresas se generan cambios que inciden en la relación de los tres elementos mencionados antes, por lo tanto se hace necesario identificar el efecto de los cambios en cada una de las variables, la relación de mutua dependencia permite que la variación en cualquiera de las variables traiga efectos sobre las demás; en este sentido para la gerencia es relevante controlar aquello que afecte la utilidad.

El estudio evidenció que en estas empresas los productos fabricados en las diversas líneas alcanzan un costo promedio de producción que no superan los treinta mil pesos; sobre esta base se fija el precio de venta de los mismos, sin perder de vista los precios de la competencia. Por todo esto, cuando existan diferencias significativas entre unos y otros, se hace necesario ajustar costos y precios para ser competitivo, respecto a dichos factores de manera que sea posible mantenerse en el mercado. La Figura 3 muestra los costos promedios de producción; en ella se observa que un número representativo de empresas manejan el costo promedio de $\$ 15.000$ y $\$ 30.000$ pesos moneda corriente; este valor responde a un costeo total en el que los costos fijos y variables se integran al costo del producto.

\section{Gráfica 3. Costos promedio de producción}

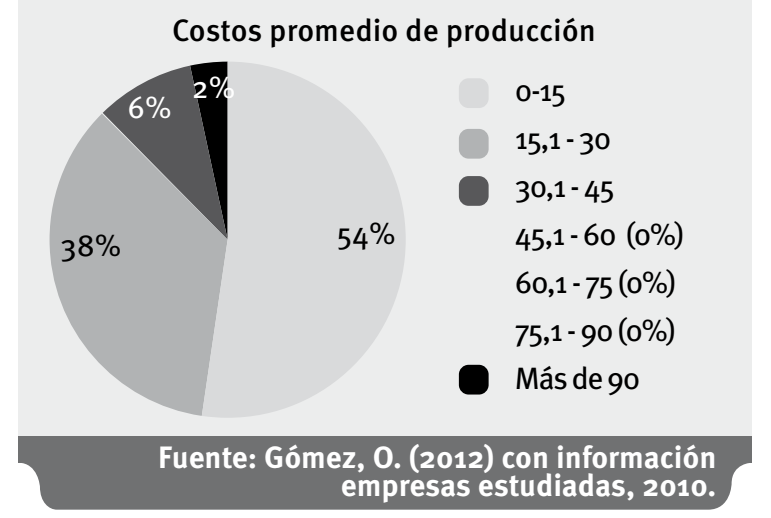

Como se dijo antes, la dinámica de los costos guarda una estrecha relación con los procesos de producción, por lo tanto la estrategia de costos se debe orientar con la minimización de los mismos a través de la optimización de recursos, con miras a lograr el aumento de la productividad medida por el incremento en el nivel de operación. 
Otro aspecto sobre el cual se direccionan las estrategias en esta industria, corresponde a los precios de venta, estos tiene relación directa con el costo, es decir que a menores costos, menores precios y mayores posibilidades de competir en el mercado. Vale la pena reconocer, que los precios de venta que fijan a los productos fabricados oscilan entre $\$ 20.000$ y $\$ 40.000$, valores relativamente bajos respecto al contenido artesanal que agregan al producto; es decir que a pesar de poseer alto componente manual, los precios se mantienen. El $89 \%$ de estas empresas, muestran que el precio de venta de los productos no supera los $\$ 40.000$ pesos moneda corriente (Gráfica 4 ).
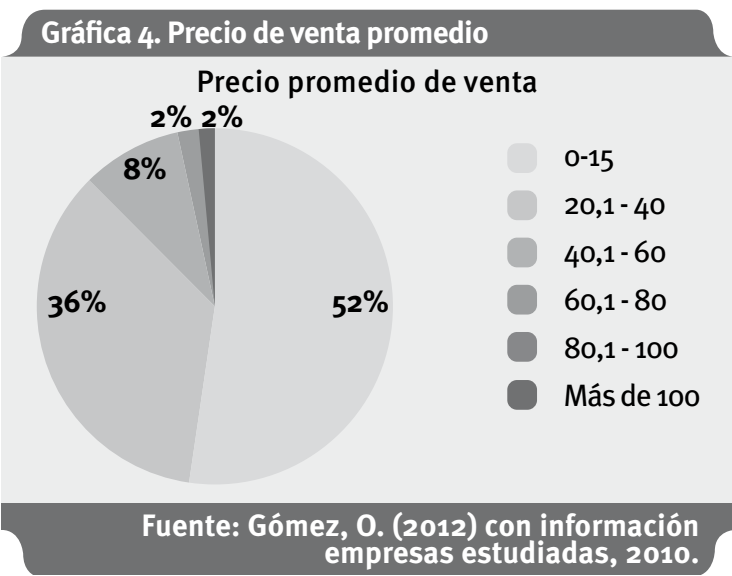

Queda definido que costo y precio de venta no pueden estar separados, el uno va con el otro y la relación entre ellos genera el margen de contribución. Es preciso señalar que esta industria aplica la estimación del costo de producción para una muestra y sobre este fija el precio de venta con el cual sale al mercado; por lo tanto, se hace necesario tener especial cuidado en la estimación para no afectar el desempeño financiero de la organización con los costos de fabricación y venta en la producción real.

Por todo lo expuesto hasta aquí, es pertinente examinar lo relacionado con el margen de contribución, de este depende la utilidad de la organización que se refleja en el estado de resultados de las operaciones de un período. La gerencia define un margen de contribución suficiente para cubrir los costos del período y que a su vez se ajuste a los precios del mercado; cualquier decisión que se tome al respecto sin la suficiente evaluación económica y financiera, puede llegar a afectar la ventaja que poseen estas organizaciones frente a los competidores. La Figura 7 muestra los elementos que son tenidos en cuenta por la industria de confecciones en función del margen de contribución y la utilidad.
Definitivamente, esta industria determina el margen de contribución y la utilidad en función del volumen de producción y minimización de costos. Todo esto indica que las estrategias giran alrededor de estas dos variables con el fin de controlarlas y al mismo tiempo se conviertan en opciones estratégicas para soportar el desempeño financiero y proyecte a estas organizaciones a la búsqueda del posicionamiento por diferenciación, calidad, precio y liderazgo en costos totales bajos. Como respuesta a los resultados obtenidos de estas organizaciones, en lo relacionado con la dinámica de costos, estrategias y formas de producción, fue posible iniciar una segunda fase de investigación con el fin de intervenir la problemática evidenciada, con un sistema de costos ajustado con las condiciones de estas empresas y apoyado en una herramienta tecnológica que dinamice y controle la producción, al mismo tiempo contribuya con la gestión de costos para la toma de decisiones, en las micro y pequeñas empresas industriales de confección textil.

\section{Conclusiones}

El análisis realizado en las empresas de la industria de confecciones infantiles de Bucaramanga, permitió establecer que $88 \%$ de estas, son micro y pequeñas empresas, que trabajan en condiciones estrechas al no contar con suficientes recursos para el desarrollo de las operaciones. Sin embargo, poseen ventajas competitivas, sobre todo en diseño, especificaciones y calidad de las materias primas utilizadas, mano de obra calificada y el componente artesanal, atributos que les ha permitido alcanzar reconocimiento y posesionar los productos en el mercado regional, nacional e internacional.

De igual forma se logró establecer que las estructuras de costos son un poco incipientes, aunque integran al producto la totalidad de los costos; la metodología con la que lo realizan se basa sobre todo en la experiencia. Sin embargo, se evidencia que existe una estrecha relación entre los costos, con el volumen de producción y la utilidad; a su vez esta relación permite la fijación del precio de venta y margen de contribución. El estudio permitió determinar que los costos son base fundamental para fijar el precio de venta; $31 \%$ de estas empresas fijan el precio con base en costos estimados y el $23 \%$ de ellas se soportan en costos estimados y la competencia.

De otra parte, los costos promedio de producción en un $54 \%$ llegan hasta $\$ 15.000$ que es un valor relativamente bajo, en $38 \%$ de la muestra observada sus costos se encuentran en un intervalo entre $\$ 15.000$ 
y $\$ 30.000$, es decir que en un alto grado los costos de producción de estas empresas se clasifican en rango bajo y medio. En menor proporción manejan costos relativamente altos, esto también dependen del tipo de producto que fabrican y del mercado en el que participan. Igualmente, fue posible identificar que el precio se fija en función del costo y la competencia; por lo tanto $52 \%$ de estas empresas manejan un precio promedio de hasta $\$ 20.000$ y el $36 \%$ fijan precios de venta en un rango de $\$ 20.000$ a $\$ 40.000$, equiparados con la competencia.

Finalmente, el análisis de las variables relacionadas con costos de producción, precio de venta y margen de contribución, permitió establecer que las estrategias las enfoca a la minimización de costos a través de la productividad, con miras a elevar el nivel de producción y manteniendo la calidad. De ahí que se formulen estrategias en busca de la productividad; el $44 \%$ busca la productividad mediante la capacitación para mejorar la curva de aprendizaje, el $29 \%$ mediante el uso de tecnología y el $21 \%$ por medio de la asociatividad, para compartir experiencia y recursos. Definitivamente el margen de contribución se soporta en volumen de producción ( $27 \%$ ), minimización de costos $(21 \%)$ y en volumen de producción y costos (31\%); esto indica que las estrategias son definidas en función de estos componentes, con el fin de apoyar el fortalecimiento de la relación costo-volumen-utilidad, para fomentar el desempeño financiero y la sostenibilidad de estas empresas en el mercado.

\section{Referencias}

Amaya, J. (2005). Gerencia, planeación y estrategia. Bucaramanga, Colombia: Universidad Santo Tomás (pp. 34-35).

Asociación Nacional de Empresarios de Colombia (ANDI). (2009). Informe ANDI. Santafé de Bogotá, Colombia: ANDI.

Barker, R. (2005). Reportes de desempeño financiero. Revista Internacional Legis de Contabilidad \& Contaduría, 20, (pp. 89-122).

Cámara de Comercio. (2010). Base de datos empresas industria de confecciones infantiles. Bucaramanga, Colombia: Cámara de Comercio.

Cámara de Comercio. (2010). Cámara directa. Bucaramanga, Colombia: Cámara de Comercio.
Cámara de Comercio (2010). Indicadores económicos. Bucaramanga, Colombia: Cámara de Comercio.

Deming, E. (1989). Calidad, productividad y competitividad. La salida de la crisis. Madrid, España: Díaz de Santos (p. 9).

Faga, H., y Ramos, M. (2007). Como conocer y manejar sus costos para tomar decisiones rentables. Buenos Aires, Argentina: Editorial Ediciones Granica S.A. (p.12).

Hargadon, B., y Múnera, A. (2003). Contabilidad de costos. Santafé de Bogotá, Colombia: Grupo Editorial Norma (p. 95).

Horngren, C., Datar, S., y Foster, G. (2007). Contabilidad de costos. Un enfoque gerencial (XII ed.). México, DF, México: Editorial Pearson, Prentice Hall (p. 6o).

Kim, R., y Mauborgne, R. (2005). La estrategia de Océano Azul. Bogotá, Colombia: Editorial Norma (p.23).

León, O. (1999). Administración financiera. Fundamentos y aplicaciones. Cali, Colombia: Editorial Prensa Moderna Impresores S.A. (p. 2).

Martínez, C. (2007). Estadística y muestreo. Bogotá, Colombia: Editorial ECOE EDICIONES (pp. 390-392).

Meléndez, H. (2004). Gestión de producción. Bucaramanga, Colombia: Universidad Santo Tomás (p. 15).

Méndez, C. (2005). Metodología. Diseño y desarrollo del proceso de investigación. México, DF, México: Editorial Mc Graw Hill (p. 136).

Polimeni, R., Fabozzi, F., y Adelberg, A. (2005). Contabilidad de costos. Conceptos y aplicaciones para la toma de decisiones gerenciales. Bogotá, Colombia: Editorial McGraw Hill (p. 11).

Porter, M. (2007). Estrategia competitiva. México, DF, México: Grupo Editorial Patria (pp. 13-51).

Ramírez, D. (2008). Contabilidad administrativa. México, DF, México: Editorial McGraw Hill (p.69).

Sinisterra, G. (2006). Contabilidad de costos. Bogotá, Colombia: Editorial ECOOE EDICIONES (p. 178).

Trujillo, M. (2007). Informe ACOPI Sant. Bucaramanga, Colombia: Asociación Nacional de Pequeños Industriales ACOPI, Santander. 